\title{
Electrochemical Immobilisation of Enzymes
}

\author{
Part 4. - Co-immobilisation of Glucose Oxidase and Ferro/Ferricyanide in \\ Poly(N-methylpyrrole) Films $\dagger$
}

\author{
P. N. Bartlett, ${ }^{*}$ Z. Alit and V. Eastwick-Field \\ School of Chemistry, University of Bath, Bath BA4 7AL, UK
}

\begin{abstract}
Glucose oxidase and ferro/ferricyanide can be co-immobilised in poly( $\mathrm{N}$-methylpyrrole) films. The two species compete for incorporation into the films so that on increasing the concentration of enzyme in the growth solution less ferro/ferricyanide is incorporated into the film, and vice versa. The ferro/ferricyanide entrapped within the film acts as a redox mediator for the oxidation of the enzyme at $0.45 \mathrm{~V} v \mathrm{~s}$. SCE. Studies of the effect of variation of film thickness, glucose concentration, enzyme loading and ferro/ferricyanide loading are consistent with a model in which diffusion and enzyme-catalysed reaction within the film are coupled. Analysis of data from these experiments allows the rate parameters for these processes to be determined. These data are compared with previous results for oxygen mediation in poly $(N$-methylpyrrole) films.
\end{abstract}

Electropolymerisation is a flexible and readily controlled method for the immobilisation of enzymes at electrode surfaces. It has the advantage over conventional covalent immobilisation that the thickness and spatial extent of the immobilised enzyme layer can be controlled. This makes electropolymerisation an attractive method for the immobilisation of enzymes on microelectrode structures and for bioelectronic applications.

A number of studies of enzyme electrodes based on the immobilisation of glucose oxidase (GOx) in conductingpolymer films have been published. Glucose oxidase catalyses the oxidation of glucose in a two-step process. In the first step, the oxidised form of the enzyme GOx(FAD) reacts with glucose to produce the product gluconolactone and the reduced enzyme, GOx(FADH $\left.{ }_{2}\right)$.

$\beta$-D-glucose + GOx(FAD) $\rightarrow$

$$
\text { gluconolactone-GOx(FADH } \left.{ }_{2}\right)
$$

In the second step the reduced form of the enzyme is reoxidised either by oxygen, its natural redox partner, or by some suitable artificial redox mediator, $\mathrm{M}^{+} / \mathrm{M}$.

$$
\mathrm{GOx}\left(\mathrm{FADH}_{2}\right)+\mathrm{O}_{2} \rightarrow \mathrm{GOx}(\mathrm{FAD})+\mathrm{H}_{2} \mathrm{O}_{2}
$$

or

$$
\mathrm{GOx}\left(\mathrm{FADH}_{2}\right)+2 \mathrm{M}^{+} \rightarrow \mathrm{GOx}(\mathrm{FAD})+2 \mathrm{M}
$$

Various conducting polymers have been used to immobilise the enzyme at an electrode surface including poly (pyrrole),${ }^{1-9}$ poly(indole),${ }^{10}$ poly(aniline),${ }^{11-13} \operatorname{poly}(N$ methylpyrrole $)^{14}$ and copolymers of $N$-substituted poly(pyrroles) ${ }^{15}$ electropolymerised in the presence of the enzyme. These have used either molecular oxygen ${ }^{1,2,6,7}$ or an artificial mediator ${ }^{3,5,8,9}$ such as ferrocene monocarboxylic acid or a quinone to reoxidise the enzyme.

The precise mechanism of the reactions within the conducting-polymer film and the effect of the immobilisation on the enzyme kinetics are of interest. In principle, the enzyme could be reoxidised by direct interaction with the polymer, or by reaction with mediator, or oxygen. In the latter case the reduced mediator, or hydrogen peroxide, generated by the enzyme could be oxidised within the polymer

\footnotetext{
$\dagger$ Part 3: Ref. 26

$\ddagger$ Present address: Department of Pharmacy, Brighton Polytechnic, Brighton, BN2 4GJ.
}

or at the electrode surface. In a previous $\operatorname{paper}^{14}$ we presented the results of a detailed study of GOx immobilised in poly $(N$-methylpyrrole) films with oxygen as the mediator. This work showed that the hydrogen peroxide generated by the enzyme was unreactive within the polymer film and was only detected by oxidation at the underlying platinum electrode surface. Bélanger et al. ${ }^{6}$ have reached a similar conclusion from their studies of the poly(pyrrole)-GOx system where they have shown that the hydrogen peroxide destroys the conductivity of the polymer.

It is therefore desirable to avoid the production of hydrogen peroxide by the use of a suitable mediator. This has the added advantage that a less positive potential can be used so that there is potentially less problem from interference due to the oxidation of other species present in the solution. Ferrocene derivatives are possible mediators which can be used for this purpose and in a subsequent paper we will present results for the use of ferrocene monocarboxylic acid as a mediator to glucose oxidase immobilised in poly $(\mathrm{N}$-methylpyrrole) films. Although ferrocene monocarboxylic acid is a good mediator for GOx it is not confined within the polymer and therefore must be present in the external solution. It would be more desirable if both the mediator and enzyme could be entrapped within the polymer film.

It is known that ferro/ferricyanide can be incorporated into electropolymerised films of poly(pyrrole) and that it will remain entrapped within the films as long as the polymer is not reduced. ${ }^{16-19}$ Ferricyanide is also known to act as a mediator for the oxidation of GOx. ${ }^{20,21}$ In this paper we present results of the study of GOx immobilised in poly $(\mathrm{N}$ methylpyrrole) films containing ferro/ferricyanide. We show that the ferricyanide is entrapped and can mediate the oxidation of GOx within the film.

\section{Experimental}

\section{Solutions and Reagents}

Details of the instrumentation are given in our previous paper. ${ }^{14}$ All solutions were freshly prepared using water from a Whatman RO50 water purification system and using AnalaR-grade reagents where possible. Tetraethylammonium tetrafluoroborate, TEATFB (Aldrich), was recrystallised from methanol. $N$-Methylpyrrole was freshly distilled at reduced pressure. Stock solutions of glucose were allowed to mutarotate for $24 \mathrm{~h}$ before use. Glucose oxidase (E.C.1.1.3.4), was a gift from MediSense. Solutions were deoxygenated by 
bubbling with oxygen-free nitrogen, presaturated with water, for at least $30 \mathrm{~min}$.

\section{Enzyme Immobilisation}

Platinum electrodes were prepared by polishing with slurries of 1 and $0.3 \mu \mathrm{m}$ alumina followed by copious washing with reagent-grade water. The polymer films were grown on platinum disc electrodes (Oxford Electrodes, $0.39 \mathrm{~cm}^{2}$ ) by stepping the potential from 0.0 to $0.780 \mathrm{~V} v s$. SCE in a buffered aqueous solution containing $0.050 \mathrm{~mol} \mathrm{\textrm {dm } ^ { - 3 }} \mathrm{N}$ methylpyrrole, $0.10 \mathrm{~mol} \mathrm{dm}^{-3}$ TEATFB, $0.15 \mathrm{~mol} \mathrm{dm}^{-3}$ disodium hydrogen orthophosphate adjusted to $\mathrm{pH} 7.0$ with phosphoric acid, and various concentrations of potassium ferrocyanide and GOx. This growth solution was prepared by adding potassium ferrocyanide to a deoxygenated solution of the enzyme and buffer followed by the addition of the $N$ methylpyrrole. This was done in order to avoid the generation of ferricyanide by reaction between the ferrocyanide and oxygen present in solution. The $N$-methylpyrrole was added last to minimise loss by evaporation. Coated electrodes were washed by rotating at $9 \mathrm{~Hz}$ in buffer solution for $5 \mathrm{~min}$ before use. This was necessary in order to remove any weakly adsorbed enzyme from the electrode surface.

\section{Procedure}

Glucose responses were measured at a rotating electrode $(9$ $\mathrm{Hz}$ ) at $0.45 \mathrm{~V}$ vs. SCE. The background current was first allowed to stabilise for $1 \mathrm{~h}$, after which time it was typically 1 $\mu \mathrm{A}$. The glucose concentration was altered by adding aliquots of stock solution to a known volume of deoxygenated buffer.

\section{Experiments with Labelled GOx}

GOx labelled with radioactive tritium was a gift from $A$. E. G. Cass. The enzyme was labelled by reaction with tritiated sodium borohydride to give material with $8.2 \times 10^{6}$ counts $\mathrm{mg}^{-1} \mathrm{~min}^{-1}$. For the electropolymerisation experiments the labelled GOx was diluted with 182 equivalents of unlabelled GOx so that in the growth solutions $0.55 \%$ of the enzyme was labelled. In all cases, the total enzyme concentration was $10 \mathrm{mg} \mathrm{cm}^{-3}$. For each ferrocyanide concentration, films were grown by potential steps to $0.785 \mathrm{~V} v$ s. SCE at platinum disc electrodes and the total charge recorded, typically $0.5 \mathrm{C}$. The films were removed from the electrode by stepping the potential of the electrode to $4 \mathrm{~V}$ whilst rotating it at $16 \mathrm{~Hz}$ in background buffer. The evolution of oxygen under these conditions lifts the films from the electrode surface. The films were collected and washed. For each ferrocyanide concentration five films were combined for counting. Scintillation counting was carried out using a liquid scintillation analyser (Packard 2000CA). The scintillant was prepared by dissolving $1 \mathrm{~g}$ of 1,4-di-2-(5-phenyloxazoyl)benzene (Fisons) and $25 \mathrm{~g}$ of 2,5-diphenyloxazole (BDH) in $3 \mathrm{dm}^{3}$ of xylene and $2 \mathrm{dm}^{3}$ of Triton X-100. Each sample was counted for $10 \mathrm{~min}$.

\section{Results}

\section{Preliminary Studies}

Ferrocyanide was chosen for the growth solution in order to avoid problems caused by chemical oxidation of the $N$ methylpyrrole by ferrocyanide in the bulk solution. ${ }^{18}$ In those experiments in which the growth solution was not carefully deoxygenated before the addition of the ferrocyanide, the reproducibility of film growth was poor and the solutions were found to deteriorate over a period of $1 \mathrm{~h}$. This is due to the formation of ferricyanide in the solution produced by reaction between the ferrocyanide and the dissolved oxygen. Reproducible results were obtained when freshly prepared, deoxygenated growth solutions were used, and all results reported below are for films grown in this way. Fig. 1 shows a set of steps to different potentials for a fresh monomer solution containing GOx and ferrocyanide. For steps to potentials greater than $0.80 \mathrm{~V}$, the current is initially large but decays with time; for steps to potentials below $0.75 \mathrm{~V}$, the currents are initially small and increase slowly. In all cases adherent films are produced. These observations were found to be essentially unaltered by changes in the concentration of enzyme or ferrocyanide.

Some differences were observed in the cyclic voltammetry of the films grown at the different potentials. For films grown at potentials above $0.8 \mathrm{~V}$ the cyclic voltammograms show little oxidation or reduction of the film, suggesting that overoxidation of the polymer has occurred during growth leading to an insulating film. For films grown at potentials below $0.75 \mathrm{~V}$ the voltammetry is poorly resolved. Potentials between 0.78 and $0.80 \mathrm{~V}$ were found to represent the best compromise. For all subsequent experiments, films were grown by stepping to $0.79 \mathrm{~V}$.

Fig. 2 shows a typical cyclic voltammogram for a film grown at $0.79 \mathrm{~V}$ recorded in buffered background electrolyte solution. Comparison of cyclic voltammograms for poly $(\mathrm{N}$ methylpyrrole) films containing GOx grown with and without ferrocyanide present in the solution shows that, for the films grown in the presence of ferrocyanide, there is an additional redox process at $0.15 \mathrm{~V}$ on the anodic scan and at $0.05 \mathrm{~V}$ on the cathodic scan. The size of these peaks decreases with repeated cycling of the film between -0.2 and $0.5 \mathrm{~V}$, but they are stable when the film is held in its oxidised state. These observations are consistent with previous work on poly(pyrrole) films containing entrapped ferro/ferricyanide. ${ }^{16-19}$ This additional redox process can be associated with the oxidation and reduction of the ferro/ferricyanide entrapped within the polymer.

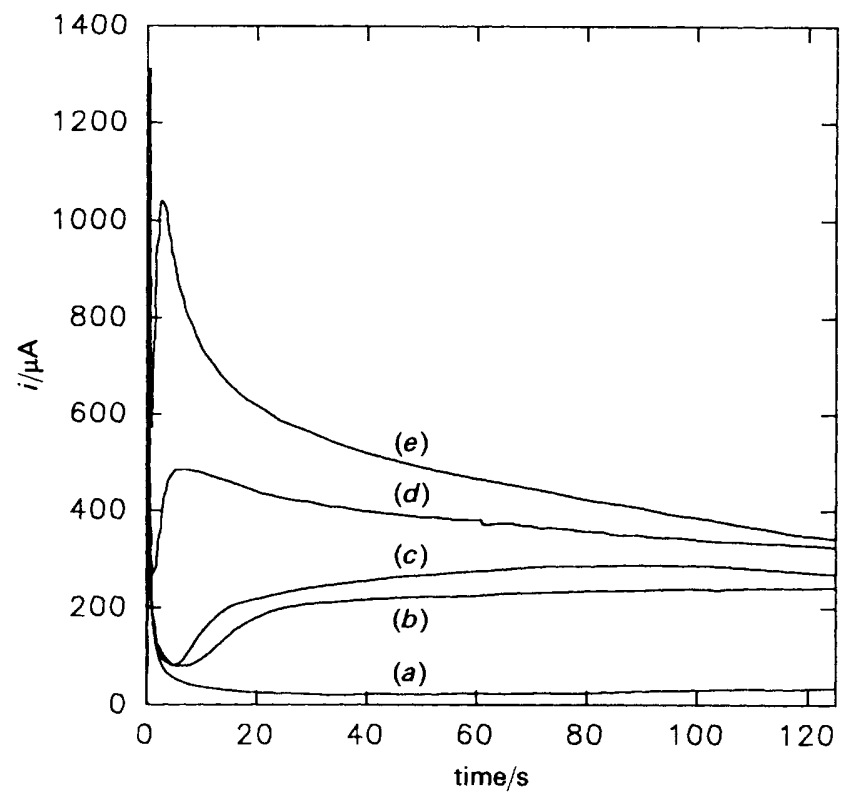

Fig. 1 A typical set of current transients recorded during the growth of poly( $N$-methylpyrrole) films containing $\mathrm{GOx}$ and ferrocyanide. The films were grown from a buffered aqueous solution pH 7.2 containing $0.15 \mathrm{~mol} \mathrm{dm}{ }^{-3}$ phosphate, $0.1 \mathrm{~mol} \mathrm{dm}^{-3}$ TEATFB, $50 \mathrm{mmol} \mathrm{dm} \mathrm{m}^{-3} \mathrm{~N}$-methylpyrrole, $5 \mathrm{mg} \mathrm{cm}^{-3} \mathrm{GOx}$ and 5 mol dm ${ }^{-3}$ ferrocyanide. Potentials (in mV vs. SCE): (a) 750 , (b) 800 , (c) $825,(d) 850,(e) 900$ 


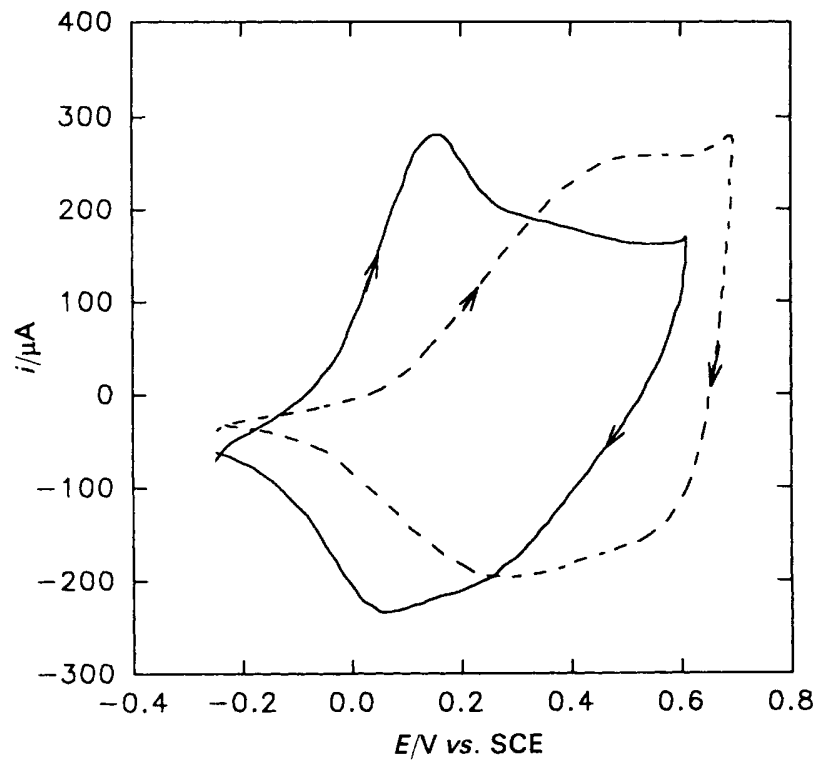

Fig. 2 Cyclic voltammograms of electrodes coated with poly $(N$ methylpyrrole) films containing either entrapped GOx (---) or entrapped GOx and ferrocyanide $(-$ ) recorded in glucose-free buffer at $20 \mathrm{mV} \mathrm{s}^{-1}$. The films were grown at $0.79 \mathrm{~V}$ from aqueous buffered solutions containing $50 \mathrm{mmol} \mathrm{dm}{ }^{-3} N$-methylpyrrole and $8.5 \mathrm{mg} \mathrm{cm} \mathrm{cm}^{-3} \mathrm{GOx}$ in either the presence or absence of $20 \mathrm{mmol}$ $\mathrm{dm}^{-3}$ ferrocyanide

We have used cyclic voltammetry to investigate the effect of the concentration of ferrocyanide in the growth solution on the amount of ferro/ferricyanide incorporated in the film. In these experiments the charge passed to grow the films was kept constant. Calculations using the integrated form of the Cottrell equation show that the contribution from ferrocyanide oxidation to the total charge passed to grow the film is never more than $5 \%$. We find that, at a constant enzyme concentration of $8.5 \mathrm{mg} \mathrm{cm}^{-3}$, for ferrocyanide con-

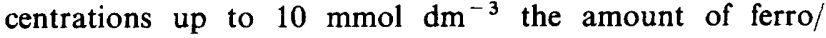
ferricyanide incorporated in the film, as determined by the anodic charge under the peak at $0.15 \mathrm{~V}$ in the cyclic voltammogram corrected for the background charge, increases linearly with increasing concentration of ferrocyanide in the growth solution, Fig. 3. We also find that at a fixed concentration of ferrocyanide and enzyme, the amount of ferro/ ferricyanide incorporated increases linearly with the total charge passed to grow the film. This is to be expected if the films are uniform.

Based on these data, and estimating the film thickness by assuming that a growth charge of $1 \mathrm{mC} \mathrm{cm}^{-2}$ corresponds to a film thickness of $0.22 \times 10^{-6} \mathrm{~cm}^{22}$ we can estimate the concentration of ferro/ferricyanide incorporated within the film. For films grown in the presence of $8.5 \mathrm{mg} \mathrm{cm}^{-3} \mathrm{GOx}$ and for concentrations of ferrocyanide in the growth solution of up to $10 \mathrm{mmol} \mathrm{dm}^{-3}$, we find that the concentration of ferrocyanide incorporated within the film is 15 times its concentration in the growth solution.

We have also investigated the effect of the variation of the enzyme concentration at a fixed concentration of ferrocyanide in the growth solution on the amount of ferro/ fericyanide incorporated in the film, Fig. 4. We find that with increasing enzyme concentration in the growth solution the amount of ferro/ferricyanide incorporated in the film decreases. Thus there appears to be a competition between the enzyme and the ferro/ferricyanide for incorporation into the film. To investigate this effect further we used radiolabelled GOx to measure the amount of enzyme incorporated in the films directly. We find that as the concentration of fer-

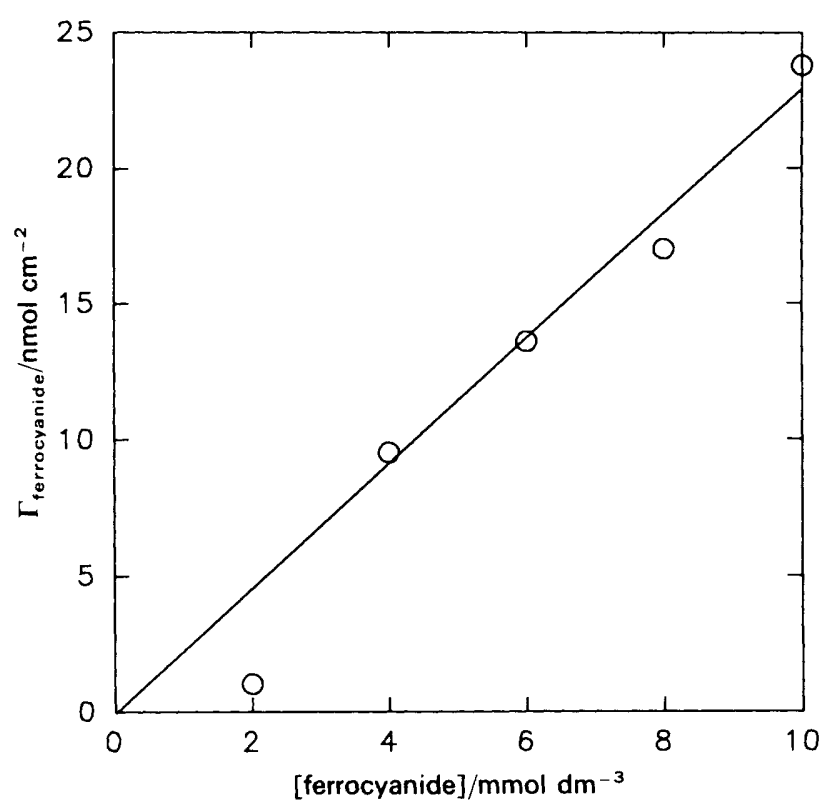

Fig. 3 A plot of the amount of ferrocyanide incorporated in the film as determined by cyclic voltammetry as a function of the concentration of ferrocyanide in the growth solution at a constant GOx concentration of $8.5 \mathrm{mg} \mathrm{cm}^{-3}$ and film thickness of $660 \mathrm{mC} \mathrm{cm}^{-2}$

rocyanide in the growth solution is increased at a fixed concentration of GOx, the amount of enzyme incorporated in the film decreases, Fig. 5. Similar results have recently been reported by Kajiya et $a .^{8}$ for poly(pyrrole) films containing entrapped GOx and hydroquinone sulfonate. In both cases it appears that the enzyme and the redox mediator, which are both negatively charged at $\mathrm{pH} \mathrm{7,} \mathrm{compete} \mathrm{to} \mathrm{balance} \mathrm{the}$ charge on the oxidised polymer. Thus the more enzyme incorporated, the less mediator, and vice versa.

In all subsequent experiments we standardised on a growth solution containing $10 \mathrm{mmol} \mathrm{dm} \mathrm{dm}^{-3}$ ferrocyanide and $8.5 \mathrm{mg}$ $\mathrm{cm}^{-3} \mathrm{GOx}$.

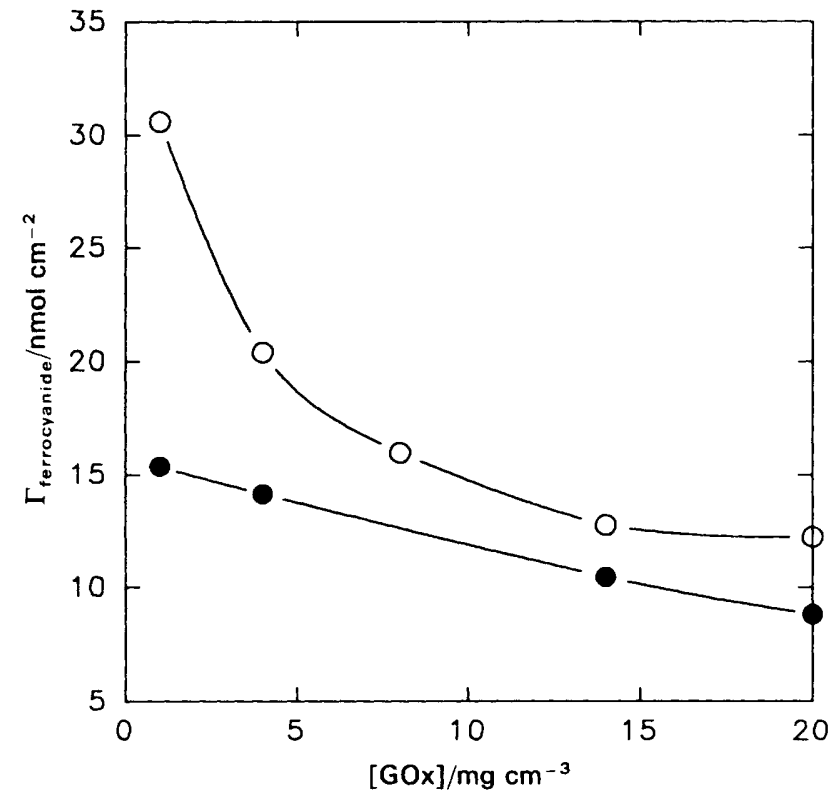

Fig. 4 A plot of the amount of ferrocyanide incorporated in the film as determined by cyclic voltammetry as a function of the concentration of GOx in the growth solution at constant concentrations of ferrocyanide of (O) 8 and (O) $15 \mathrm{mmol} \mathrm{dm}^{-3}$ and for a constant film thickness of $490 \mathrm{mC} \mathrm{cm}-2$ 


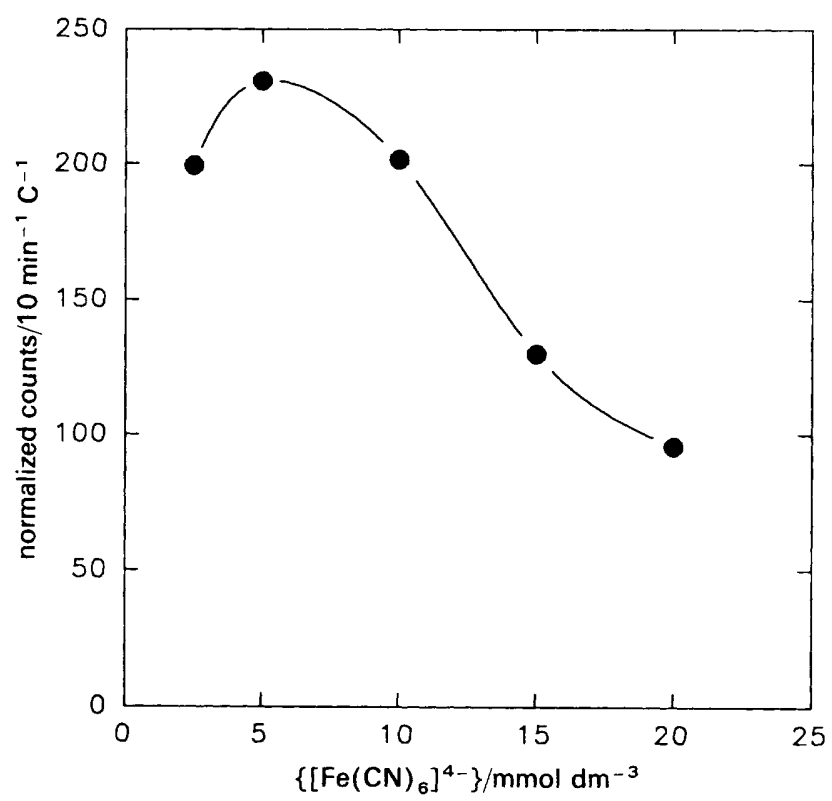

Fig. 5 Plot of the amount of tritium-labelled GOx incorporated within the film, as determined by scintillation counting and normalised with respect the charge passed to grow the film, as a function of the concentration of ferrocyanide in the growth solution. Data refer to a constant concentration of GOx in the growth solution of $10 \mathrm{mg}$ $\mathrm{cm}^{-3}$

\section{Voltammetry of Solution Species at the Coated Electrode}

Ideally we would like the polymer film to be conducting so that the mediator can react on the polymer within the film. This would then ensure rapid and efficient regeneration of the mediator after reaction with the enzyme. In order to see whether this was possible we have investigated the electrochemistry of ferrocyanide in solution at the enzyme-coated electrode. Fig. 6 shows Koutecky-Levich plots for the oxidation of ferrocyanide at a clean platinum electrode, a poly $(N$ methylpyrrole)-coated electrode and poly( $N$-methylpyrrole)-

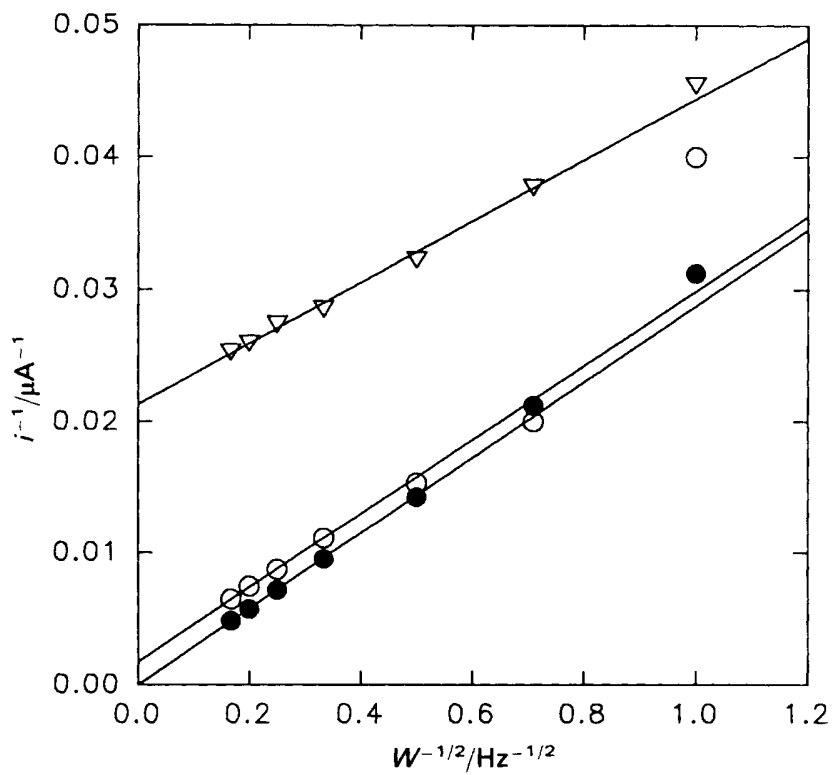

Fig. 6 Koutecky-Levich plots for the oxidation of $10 \mathrm{mmol} \mathrm{dm}{ }^{-3}$ ferrocyanide at a clean platinum electrode $(O)$, a poly $(N$-methylpyrrole)-coated electrode $(O)$ and an electrode coated with a poly $(N$ methylpyrrole) film containing entrapped GOx and ferro/ferricyanide (V) coated electrodes containing immobilised GOx and ferro/ ferricyanide. The Koutecky-Levich analysis applies to firstorder processes and essentially separates the mass-transport terms from the contributions from other rate-limiting processes. In the limiting current region, the analysis gives

$$
n F A / i=1 / k_{\mathbf{D}}^{\prime} c_{\infty}+1 / k c_{\infty}
$$

where $k_{\mathrm{D}}^{\prime}$ is the mass transport rate constant, $k_{\text {? }}$ is a potential-independent rate constant and $c_{\infty}$ is the bulk concentration of ferrocyanide.

Our results show that for the electrodes coated with poly $(N$-methylpyrrole) the oxidation of ferrocyanide from solution occurs at a mass-transport-limited rate. This is consistent with the presence of a conducting film on the electrode and the oxidation of the ferrocyanide at the outside of this film. For the electrodes coated with poly( $N$-methylpyrrole) containing entrapped ferrocyanide and GOx the KouteckyLevich plots show that there is an additional process, characterised by the rate constant $k_{\text {? }}$, which limits the current at high rotation speeds. Thus, although the poly $(N$-methylpyrrole) film itself is conducting, under the conditions of our experiment, there is a slow kinetic step which limits the rate of oxidation of solution ferrocyanide at films containing GOx and ferro/ferricyanide. It is known that hydrogen peroxide reacts with poly(pyrrole) and poly( $N$-methylpyrrole) films leading to a loss of conductivity. We believe that a similar process is occurring in our experiments caused by hydrogen peroxide generated by reaction between ferrocyanide and dissolved oxygen catalysed by GOx within the film

$$
2\left[\mathrm{Fe}(\mathrm{CN})_{6}\right]^{4-}+\mathrm{O}_{2}+2 \mathrm{H}^{+} \rightleftharpoons 2\left[\mathrm{Fe}(\mathrm{CN})_{6}\right]^{3-}+\mathrm{H}_{2} \mathrm{O}_{2} \text { (4) }
$$

We can estimate the equilibrium constant, $K$, for this reaction to be $5 \times 10^{-3}$, by taking $E_{\mathrm{O}_{2} / \mathrm{H}_{2} \mathrm{O}_{2}}^{\mathrm{O}}=0.695 \mathrm{~V}$ and $E^{0}$ for the ferro/ferricyanide within the film as $0.342 \mathrm{~V}$. Although the equilibrium lies to the left, the continuous removal of hydrogen peroxide by reaction with the polymer and consequent loss of conductivity could explain the kinetic limitation on the rate of ferrocyanide oxidation at the coated electrode.

\section{Responses to Glucose}

Fig. 7 shows a typical set of responses for a coated electrode to the addition of aliquots of glucose recorded at $0.45 \mathrm{~V} v \mathrm{~s}$. SCE. In this experiment the responses are limited by the mixing time for the glucose in solution. The response of the electrode is evidently rapid, less than $2 \mathrm{~s}$.

In order to investigate the kinetics of the reactions occurring within the film, we have studied the effects of film thickness, enzyme loading and ferrocyanide loading on the responses. We begin with the effect of film thickness. The film thickness was varied by altering the deposition time. The amount of charge passed in deposition was recorded and the thicknesses were calculated assuming that a charge of $1 \mathrm{mC}$ $\mathrm{cm}^{-2}$ corresponds to a thickness of $0.22 \times 10^{-6} \mathrm{~cm}$. This figure is taken from ellipsometric studies of the deposition of poly( $N$-methylpyrrole) from aqueous buffer solutions containing GOx. ${ }^{22}$ These studies showed that the film thickness was linearly related to the charge for films of up to at least $150 \mathrm{~nm}$ thickness. The estimate of thickness may be slightly high in the present case since it does not allow for the charge passed in the oxidation of the ferrocyanide; however, this contribution is small vide supra.

In those cases in which the platinum electrode was left in contact with the growth solution for $5 \mathrm{~min}$ before the start of the polymer deposition, the responses increase with decreasing film thickness, Fig. 8. This is caused by adsorption of the enzyme onto the platinum surface leading to enhanced currents for the thin films. ${ }^{14}$ Szucs et al. ${ }^{23}$ have reported 


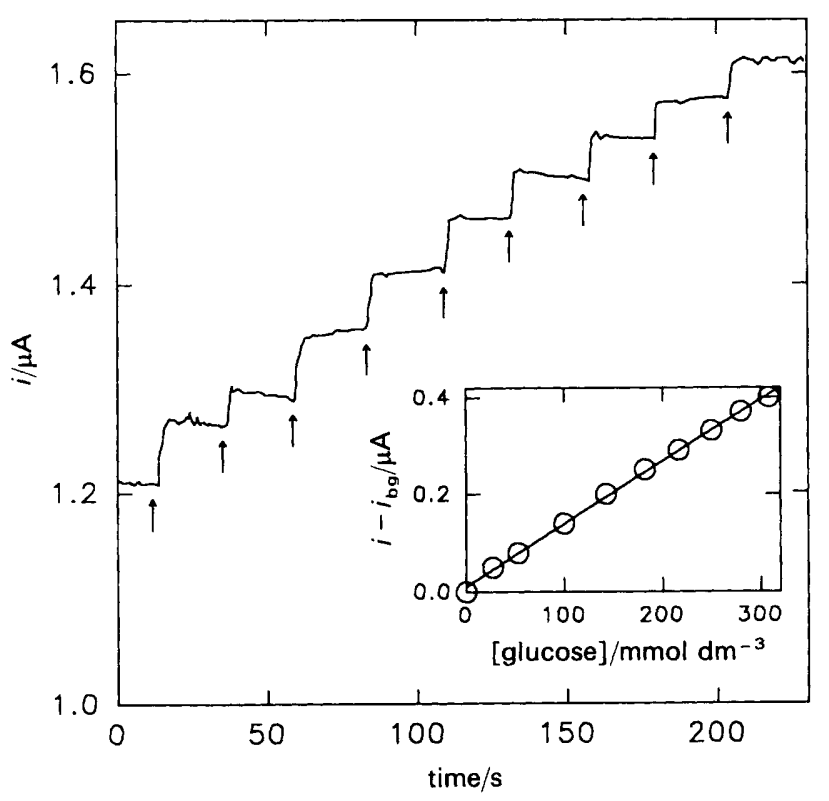

Fig. 7 Plot of the responses to glucose: the film was grown from a solution containing $10 \mathrm{mmol} \mathrm{dm}{ }^{-3}$ ferrocyanide and $8.5 \mathrm{mg} \mathrm{cm}^{-3}$ GOx without pre-adsorption of the enzyme. The electrode was held at $0.45 \mathrm{~V}$ vs. SCE during the measurement in deoxygenated buffer. Each arrow corresponds to the addition of an aliquot of glucose. The inset shows a typical plot of the current, corrected for the background current, $i_{\mathrm{bg}}$, as a function of the glucose concentration

studies of the adsorption of GOx on gold electrodes using ellipsometry. When the growth of the film is initiated immediately the electrode is placed in solution, this effect disappears and the responses decrease with decreasing film thickness, Fig. 8. In both cases, the response is independent of film thickness for the thicker films. We can explain this behaviour as follows. ${ }^{24}$ For the thin films, grown without pre-adsorption of enzyme at the electrode surface, the response to glucose increases as the film thickness increases. This is because the reaction is occurring throughout the film

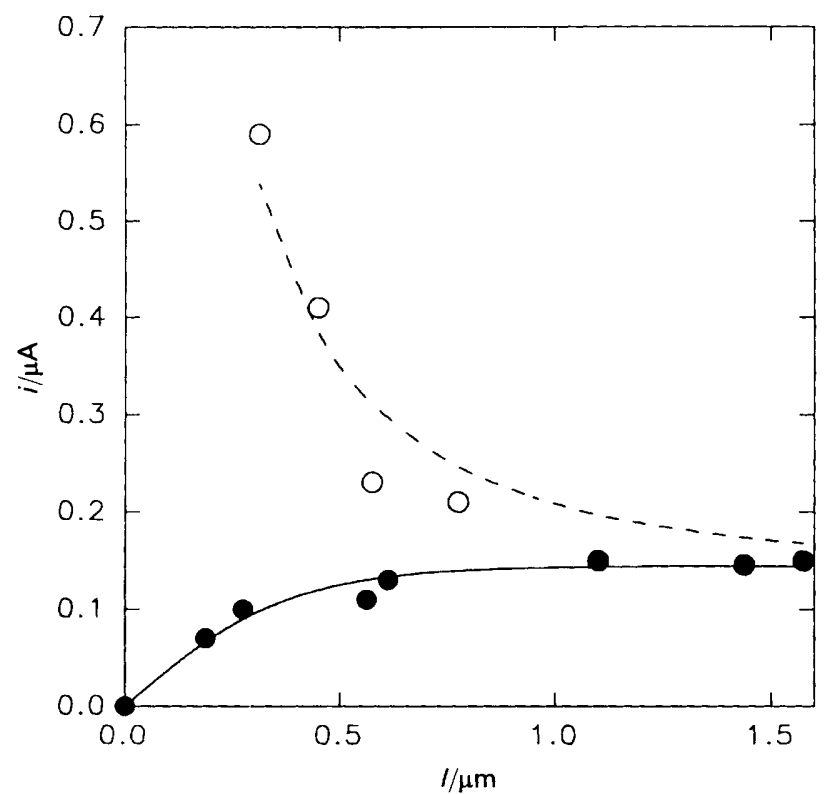

Fig. 8 A plot of the response recorded at $+0.45 \mathrm{~V}$ for $100 \mathrm{mmol}$ $\mathrm{dm}^{-3}$ glucose as a function of the film thickness showing the effect of pre-adsorption of the enzyme onto the platinum electrode surface. Films were grown from solutions containing $10 \mathrm{mmol} \mathrm{dm}^{-3} \mathrm{fer}$ rocyanide. (O) Pre-adsorbed GOx, (O) no pre-adsorption and so as the film thickness increases more enzyme is available to react with the glucose. For the thick films, the glucose is consumed in a first-order reaction layer at the polymer/ electrolyte interface and does not penetrate throughout the film. Thus increasing the film thickness has no effect on the response. Note that the behaviour differs from that with oxygen as a mediator ${ }^{14}$ because, in this case, the mediator species is entrapped within the film. From the analysis in our previous paper we find that the current under these conditions is given by [eqn. (16) of ref. (24)]

$$
j_{\mathrm{s}}=D_{\mathrm{s}} K_{\mathrm{s}} s_{\infty} \tanh \left(l / X_{\mathrm{k}}\right) / X_{\mathrm{k}}
$$

where $j_{\mathrm{s}}$ is the flux, $D_{\mathrm{s}}$ and $K_{\mathrm{s}}$ are the diffusion and partition coefficients for the glucose in the film, $l$ is the film thickness, $X_{\mathrm{k}}=\left(D_{\mathrm{s}} K_{\mathrm{M}} / k_{\mathrm{cat}} \xi[\mathrm{ez}]\right)^{1 / 2}$ is the kinetic length, [ez] is the concentration of enzyme in the growth solution in $\mathrm{mg} \mathrm{cm}^{-3}$, $\xi[\mathrm{ez}]$ is the enzyme concentration within the film and $K_{\mathrm{M}}$ and $k_{\text {cat }}$ describe the kinetics of the reaction between the enzyme in the film and glucose. When the enzyme is pre-adsorbed on the platinum surface, the current is much larger for the thin films because of the greater amount of enzyme available. For the thick films, the glucose is all consumed at the outside of the film and so the pre-adsorbed enzyme has no effect.

From the best fit of the data in Fig. 8 (obtained without pre-adsorption of the enzyme) to eqn. (II) we obtain estimates of the values of $D_{\mathrm{S}} K_{\mathrm{s}}$ and $D_{\mathrm{S}} K_{\mathrm{M}} / k_{\mathrm{cat}} \xi$ of $7.5 \times 10^{-13} \mathrm{~cm}^{2}$ $\mathrm{s}^{-1}$ and $1.2 \times 10^{-8} \mathrm{mg} \mathrm{cm}^{-1}$. It is instructive to compare these values with those obtained in our previous work on GOx immobilised in poly( $N$-methylpyrrole) films using oxygen as the mediator. ${ }^{14}$ The value for $D_{\mathrm{s}} K_{\mathrm{s}}$ obtained in the present case is $c a .20$ times smaller. This difference probably reflects differences in the structure of the polymer as grown under the two different sets of conditions. Turning to the value for $D_{\mathrm{S}} K_{\mathrm{M}} / k_{\text {cat }} \xi$ and comparing films grown with and without ferrocyanide present, we find that $D_{\mathrm{S}} K_{\mathrm{M}} / k_{\text {cat }} \xi$ is $c a$. 45 times larger for the films containing ferrocyanide. In part, this is because the presence of ferrocyanide in the growth solution means that there is less enzyme incorporated into the film; from Fig. 5, this accounts for a factor of $c a$. 2. The remaining difference must then arise from differences in $D_{\mathrm{S}} K_{\mathrm{M}} / k_{\text {cat }}$ for the two films.

Turning to the effect of the glucose concentration, eqn. (II) predicts a linear relationship between the concentration and the response. This is indeed observed; the inset in Fig. 7 shows a typical calibration for a film grown without preadsorption of GOx. The response continues to increase even

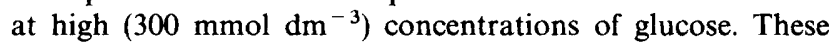
values are well above the Michaelis constant, $K_{\mathrm{M}}$, of 33 mmol dm ${ }^{-3}$ for GOx in homogeneous solution. ${ }^{25}$ This result is consistent with our previous work on the $\mathrm{GOx}-\operatorname{poly}(N$ methylpyrrole) system with oxygen as the redox mediator ${ }^{14}$ and arises because of the low partition coefficient for glucose, $K_{\mathrm{s}}$, into the polymer. From the slope of the glucose calibration curve we obtain a second estimate of $D_{\mathrm{s}} K_{\mathrm{s}}$ of $7.0 \times 10^{-13} \mathrm{~cm}^{2} \mathrm{~s}^{-1}$. This agrees well with the value estimated from the film-thickness experiments.

For the thick films, where $l>X_{\mathbf{k}}$, eqn. (II) reduces to

$$
j_{\mathrm{s}}=K_{\mathrm{s}} s_{\infty}\left(D_{\mathrm{s}} k_{\mathrm{cat}} \xi[\mathrm{ez}] / K_{\mathrm{M}}\right)^{1 / 2}
$$

so that the response should be dependent on the square root of the enzyme loading. Fig. 9 shows a plot of the response against the square root of the enzyme concentration in the growth solution for a thick film. From the slope of this plot, we obtain a value for $K_{\mathrm{s}}\left(D_{\mathrm{s}} k_{\mathrm{cat}} \xi / K_{\mathrm{M}}\right)^{1 / 2}$ of $6.3 \times 10^{-9} \mathrm{mg}^{-1 / 2}$ $\mathrm{cm}^{5 / 2} \mathrm{~s}^{-1}$. This is in excellent agreement with the value of $6.8 \times 10^{-9} \mathrm{mg}^{-1 / 2} \mathrm{~cm}^{5 / 2} \mathrm{~s}^{-1}$ obtained from a study of the film thickness. 


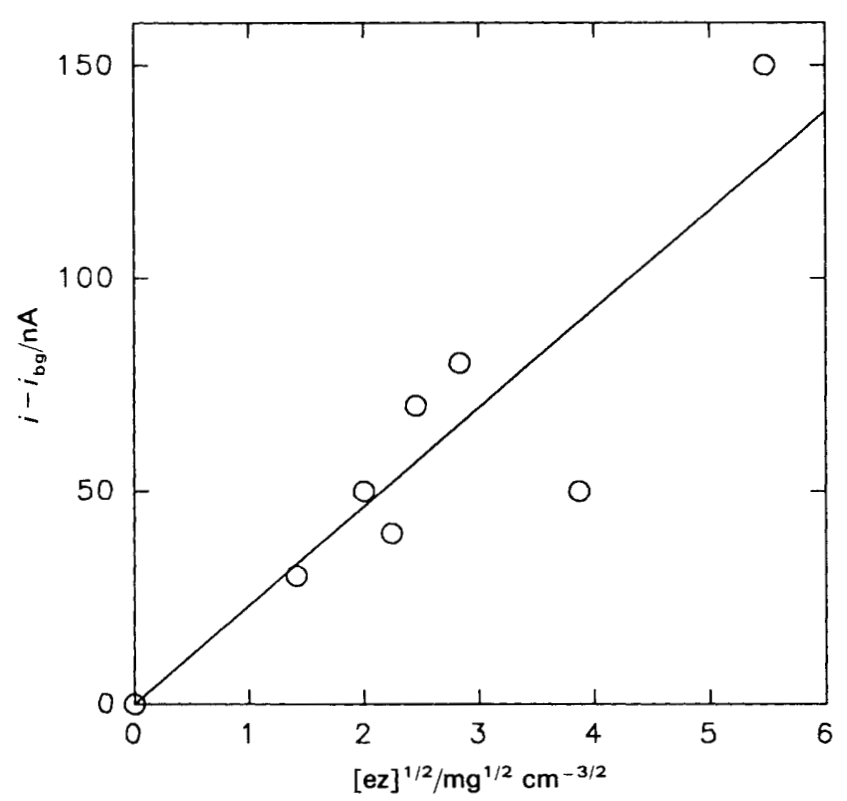

Fig. 9 Plot of the response to $50 \mathrm{mmol} \mathrm{dm}^{-3}$ glucose as a function of the square root of the concentration of enzyme in the growth solution. All films were grown from solutions containing $10 \mathrm{mmol} \mathrm{dm}^{-3}$ ferrocyanide, with a constant film thickness of $470 \mathrm{mC} \mathrm{cm}^{-2}$

Finally, we note that eqn. (II) predicts that the response is independent of the concentration of ferrocyanide incorporated within the film and this is indeed observed in our experiments, Fig. 10.

\section{Stability}

We have carried out some preliminary studies of the stabilities of our immobilised enzyme films over a two day period. We find that there is little change in the response with repeated use over $3 \mathrm{~h}$, but the responses decrease to $68 \%$ of the initial value after storage for $24 \mathrm{~h}$ at room temperature in glucose-free buffer solution.

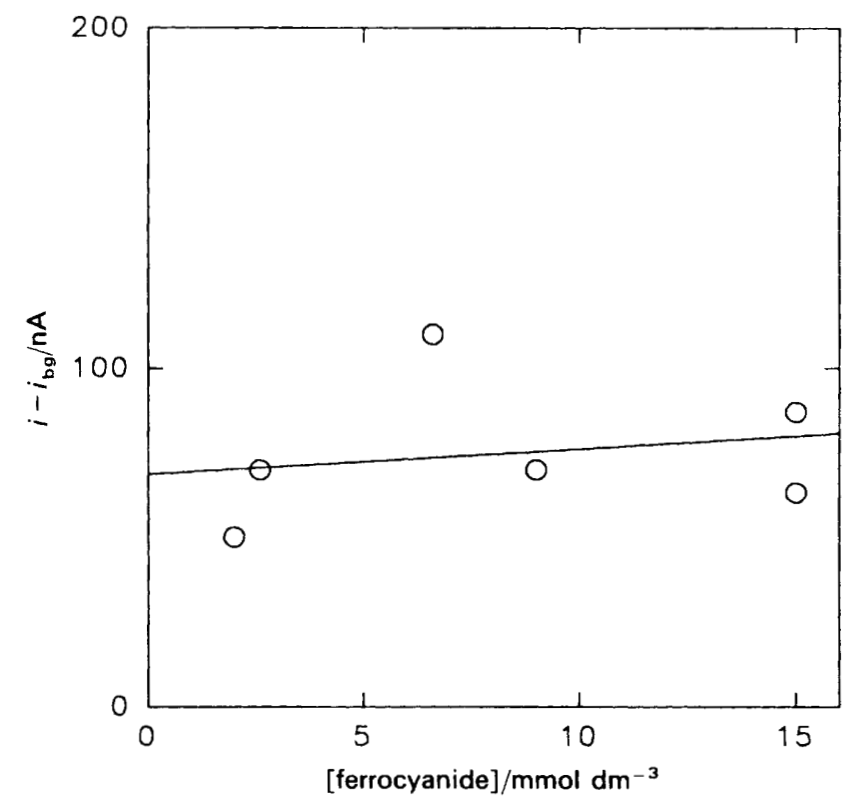

Fig. 10 Plot of the response to $53 \mathrm{mmol} \mathrm{dm}{ }^{-3}$ glucose as a function of the concentration of ferrocyanide in the growth solution. Data are for thick films, grown from solution containing $8.5 \mathrm{mg} \mathrm{cm}^{-3} \mathrm{GOx}$
Table 1 Kinetic parameters for GOx immobilised in $\operatorname{poly}(N$ methylpyrrole) films

\begin{tabular}{|c|c|c|c|}
\hline experiment & $\frac{D_{\mathrm{s}} K_{\mathrm{s}}}{/ \mathrm{cm}^{2} \mathrm{~s}^{-1}}$ & $\begin{array}{c}\left(D_{\mathrm{s}} K_{\mathrm{M}} / k_{\mathrm{cat}} \xi\right) \\
/ \mathrm{mg} \mathrm{cm}^{-1}\end{array}$ & $\begin{array}{l}K_{\mathrm{s}}\left(D_{\mathrm{s}} k_{\text {cat }} \xi / K_{\mathrm{M}}\right)^{1 / 2} \\
/ \mathrm{mg}^{-1 / 2} \mathrm{~cm}^{5 / 2} \mathrm{~s}^{-1}\end{array}$ \\
\hline \multicolumn{4}{|c|}{ ferro/ferricyanide mediation: } \\
\hline vary thickness & $7.5 \times 10^{-13}$ & $1.2 \times 10^{-8}$ & $6.8 \times 10^{-9}$ \\
\hline vary glucose & $7.0 \times 10^{-13}$ & - & - \\
\hline vary $[\mathrm{ez}]$ & - & - & $6.3 \times 10^{-9}$ \\
\hline \multicolumn{4}{|c|}{$\mathrm{O}_{2}$ mediation from ref. 14 : } \\
\hline vary thickness & $3.4 \times 10^{-11}$ & $4.8 \times 10^{-10}$ & $1.6 \times 10^{-6}$ \\
\hline vary $[\mathrm{ez}]$ & $0.4 \times 10^{-11}$ & $1.8 \times 10^{-10}$ & $0.3 \times 10^{-6}$ \\
\hline
\end{tabular}

\section{Conclusions}

Both GOx and ferro/ferricyanide can be entrapped within poly $(N$-methylpyrrole) films. Using radio-labelled GOx we are able to measure the amount of enzyme present within the film directly. Using cyclic voltammetry we can measure the amount of ferro/ferricyanide incorporated during film growth. These experiments show that the two species compete with each other for sites within the film. This is to be expected if they are both incorporated as counter anions during the deposition of the cationic polymer.

Studies of the electrochemistry of the polymer films show that at those films containing both glucose oxidase and ferro/ ferricyanide there is a slow kinetic step in the oxidation of solution ferrocyanide under the conditions used in our experiments to obtain stable reproducible responses to glucose. This is surprising because films of poly $(N$-methylpyrrole) itself, grown under similar conditions, are conducting. It is suggested that this slow kinetic step is the result of hydrogen peroxide production within the films catalysed by the entrapped enzyme. We have carried out more detailed studies of this process and these will be reported in a subsequent paper. Nevertheless, the ferro/ferricyanide remains entrapped within the film and is able to mediate the oxidation of the enzyme so that glucose can be detected amperometrically using these films at $0.45 \mathrm{~V} v s$. SCE.

The behaviour of these entrapped enzyme films is sufficiently stable and reproducible from film to film that we are able to carry out kinetic studies of the GOx-catalysed reaction within the film. By varying the film thickness, glucose concentration, enzyme loading and ferro/ferricyanide concentration, we are able to show that, for the thick films, the reaction occurs within a first-order reaction layer at the polymer/electrolyte interface and that it is not limited by the rate of re-oxidation of the enzyme by the ferricyanide. The excellent agreement between the rate parameters derived from a variety of different experiments, Table 1 , is pleasing and justifies our model. Comparison of these results with similar data for oxygen mediation shows that the presence of ferrocyanide in the deposition solution changes the properties of the film so that the product of the diffusion and partition coefficients for glucose into the films is reduced by a factor of $c a$. 20. As a consequence, the currents for glucose detection at a given glucose concentration are smaller when using ferricyanide as the co-immobilised mediator.

Finally, we note that the pre-adsorption of the enzyme onto the platinum electrode surface prior to polymer growth has a dramatic effect on the magnitude of the responses for the thin films. This is because the pre-adsorption step produces a thin, highly active, layer of enzyme at the electrode surface. We have recently exploited this effect using thin poly(phenolic) films to immobilise the pre-adsorbed enzyme layer. ${ }^{26,27}$

We thank A. E. G. Cass for the gift of labelled GOx and the SERC for financial support (GR/E67108). 


\section{References}

1 M. Umaña and J. Waller, Anal. Chem., 1986, 58, 2979.

2 N. C. Foulds and C. R. Lowe, J. Chem. Soc., Faraday Trans. 1, 1986, 82, 1259.

3 C. Iwakura, Y. Kajiya and H. Yoneyama, J. Chem. Soc., Chem. Commun., 1988, 1019.

4 S. Yabuki, H. Shinohara and M. Aizawa, J. Chem. Soc., Chem. Commun., 1989, 945.

5 J. M. Dicks, S. Hattori, A. P. F. Turner and T. Yukazawa, Ann. Biol. Clin., 1989, 47, 607.

6 D. Bélanger, J. Nadreau and G. Fortier, J. Electroanal. Chem., $1989,274,143$.

7 D. Bélanger, E. Brassard and G. Fortier, Anal. Chim. Acta, 1990, 228, 311.

8 Y. Kajiya, H. Sugai, C. Iwakura and H. Yoneyama, Anal. Chem., $1991,63,51$.

9 P. Janda and J. Weber, J. Electroanal. Chem., 1991, 330, 119.

10 P. C. Pandey, J. Chem. Soc., Faraday Trans. I, 1988, 84, 2259.

11 H. Shinohara, T. Chiba and M. Aizawa, Sensors and Actuators, 1988, 13, 79.

12 P. N. Bartlett and R. G. Whitaker, Biosensors, 1987/88, 3, 359.

13 M. Shaolin, X. Huaiguo and Q. Bidong, J. Electroanal. Chem., 1991, 304, 7 .
14 P. N. Bartlett and R. G. Whitaker, J. Electroanal. Chem., 1989, 224, 37.

15 N. C. Foulds and C. R. Lowe, Anal. Chem., 1988, 60, 2473.

16 G. Lian and S. Dong, J. Electroanal. Chem., 1989, 260, 127.

17 S. Dong and G. Lian, J. Electroanal. Chem., 1990, 291, 23.

18 J. Tietje-Girault, J. M. Anderson, I. MacInnes, M. Schröder, G. Tennant and H. H. Girault, J. Chem. Soc., Chem. Commun., 1987, 1095.

19 W. Breen, J. F. Cassidy and M. E. G. Lyons, J. Electroanal. Chem., 1991, 297, 445.

20 J. Mahenc and H. Aussaresses, C. R. Acad. Sci. Paris, 1979, 289, 357.

21 J. R. Mor and R. Guanaccia, Anal. Biochem., 1977, 79, 319.

22 R. G. Whitaker, PhD Thesis, Warwick University, 1989.

23 A. Szucs, G. D. Hitchins and J. O'M. Bockris, J. Electrochem. Soc., 1989, 136, 3748

24 P. N. Bartlett and R. G. Whitaker, J. Electroanal. Chem., 1989, 224, 27.

25 B. E. P. Swoboda and V. Massey, J. Biol. Chem., 1965, 240, 2209.

26 P. N. Bartlett, P. Tebbutt and C. H. Tyrrell, Anal. Chem., 1992, 64, 138.

27 P. N. Bartlett and D. J. Caruana, Analyst (London), 1992, 117, 1287.

Paper 2/01864K; Received 8th April, 1992 